\title{
Zero-offset internal multiple modeling in the image domain
}

\author{
Claudio Guerra and Nelson Hargreaves, Petrobras
}

Copyright 2019, SBGf - Sociedade Brasileira de Geofísica

This paper was prepared for presentation during the $16^{\text {th }}$ International Congress of the Brazilian Geophysical Society held in Rio de Janeiro, Brazil, 19-22 August 2019.

Contents of this paper were reviewed by the Technical Committee of the $16^{\text {th }}$ International Congress of the Brazilian Geophysical Society and do not necessarily represent any position of the SBGf, its officers or members. Electronic reproduction or storage of any part of this paper for commercial purposes without the written consent of the Brazilian Geophysical Society is prohibited.

\begin{abstract}
In this work, we show an internal-multiple-modeling scheme acting on the postack-migrated domain. This scheme applies when prestack data is not available yet and when internal multiples are present, posing challenges to seismic interpretation. The method is computationally inexpensive, since it uses the Exploding Reflector Model and oneway propagation. We describe the theory with details of the method, and show 3D results on real data.
\end{abstract}

\section{Introduction}

Internal multiples interference with the Pre-salt reservoirs of the Santos Basin is a known long-standing problem. This interference is more pronounced in the presence of the socalled stratified salt, which is mainly composed by a sequence of halite interbedded with anhydrite, and can have a huge impact when determining volumetrics, since it affects facies classification, impedance inversion, as well as, sometimes, structural interpretation. In the Santos Basin, generation of internal multiples mainly occurs at the sea bottom and on the top salt.

Examples of successful internal multiple attenuation in the Santos Basin include different modeling methods (Hembd et al. 2010, Cypriano et al. 2015, Krugger et al. 2018, Starring et al. 2018). Some of them are data driven, while others depend on an Earth model. Nevertheless, all of them act on the prestack data and, for this reason, are computationally intensive. Figure 1 exemplifies how misleading internal multiples can be when interfering with the Pre-salt reflectors. Steep dipping events are signal, representing periodic lava flows of the igneous basement of the Santos Basin. Gentler dip events are internal multiples. More misleading than this example is when internal multiples have dips similar to those of the Pre-salt reflectors. This can lead internal multiples to be interpreted as unconformities and even DHI's, which can negatively affect exploratory targets.

In the postack case, Filpo and Tygel (1999) used a Kirchhoff method to generate surface multiples, starting from a zero-offset stacked volume. In 2003, Pica and Manin also modeled surface multiples, but having as input a depth-migrated volume as reflectivity to generate the corresponding zero-offset stack volume via the Exploding Reflector concept. They used phase-shift extrapolation with convolution with the migrated volume to finally model the surface multiples. Wang et al. (2011) expanded Pica and Manin work to model internal multiples, using picked reflectors as the reflectivity. In this work, we propose to combine Pica and Delmas's method with that of Wang. Our method applies not only to attenuate internal multiples in postack-migrated data, but also to validate interpretation in the presence of this noise. We describe the theory, details of the method, and show 3D results on real data from three different areas of the Santos Basin.

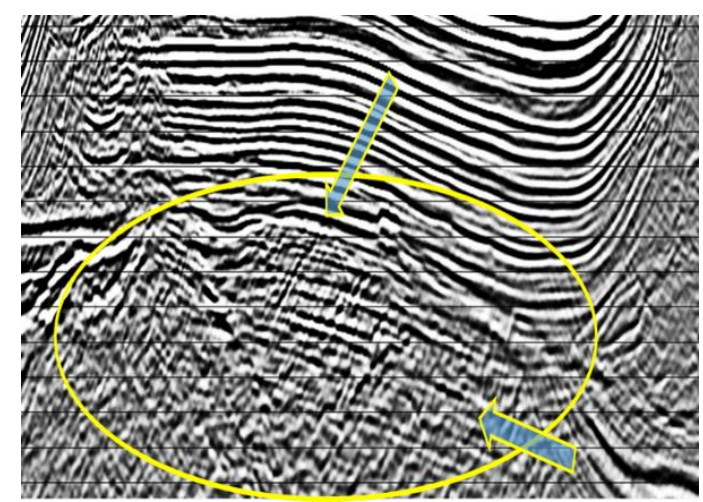

Figure 1- Steeper dip events are signal and represent periodic lava flows of the igneous basement of the Santos Basin. Gentler dip events are internal multiples.

\section{Method}

Sketch of Figure 2 shows complex trajectories zero-offset internal multiples rays can have, which naturally pushes modeling of theses multiples to wave-equation methods.

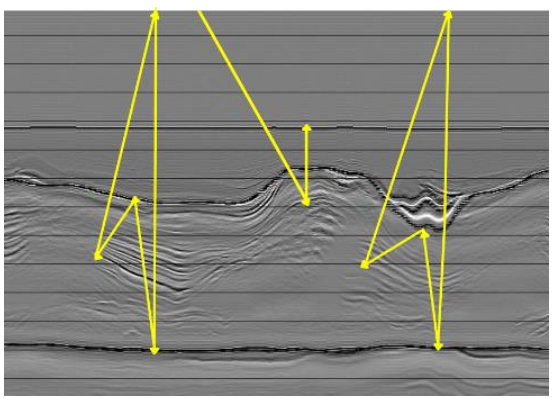

Figure 2- Possible complex trajectories of zero-offset internal multiples.

To model zero-offset internal multiples, we start with a migrated image or a better estimation of the reflectivity, a depth velocity model, preferentially that one used to produce the migrated image, and a horizon representing the internal multiple generator. We apply a sequence of propagations similar to those of wave-equation-based modeling (Pica and Delmas, 2008):

a) Zero-offset data modeling from a muted version of the reflectivity with the Exploding Reflector Concept (Lowenthal, 1976), representing primaries;

b) Downward propagation up to an auxiliary horizon, few meters below the internal multiple generator; 
c) Upward propagation, convolution with reflectivity, and storage of the result for every depth step up to the minimum depth of the generator;

d) Downward propagation, with injection of step 2, and storage of the result for every depth, down to the maximum depth;

e) Upward propagation, with injection of step 3 and convolution with reflectivity for every depth, up to the acquisition surface; and

f) Postack depth-migration of the internal multiples.

Figure 3 illustrates the modeling steps. The postackmigrated-domain internal multiples are further subtracted from the initial image. They also can de-risk interpretation, by checking if an event in the migrated image is present in the volume of the modeled multiples.

As a drawback, when the Exploding Reflector Concept fails the modelled primaries are not a good approximation of the zero-offset data and the predicted internal multiples deviate from the real ones. In addition, our method models internal multiples in the near offset only. Nevertheless, one important feature is the possibility of using different velocity models in the modeling and migration parts, which can tune in the modeled multiple.

\section{Examples}

The real data examples shown here are from 3D data of the Santos Basin, Brazil. Henceforth, the images of internal multiples are after depth migration.

The first example has the water bottom and the top of a volcanoclastic sequence as the main internal multiple generators (Figure 4a). It was applied a very aggressive adaptive summation. In Figures $4 \mathrm{~b}, \mathrm{c}$, and d, are zoomed views of the rectangle of Figure $4 a$, representing the image without multiple attenuation, the adapted multiples, and the image after attenuation, respectively. Subtraction was carried out on frequency bands. Multiple attenuation greatly improved reflector continuity in the Pre-salt.

The next example shows a clear interference of events below the salt base, which is the reflector reached by the arrow pointing down in Figure 5a. Within the oval, the steeper events correspond to signal, which are the expression of lava flows, while the gentler are internal multiples generated at the top of salt, which is the reflector on the top of the high amplitude, thicker layers. These layers are stratified salt of different composition, whose reflections bounce back at the top salt, giving rise to internal multiples. Figure $5 \mathrm{c}$ shows the result of subtracting the modeled internal multiples generated at the top salt (Figure $5 \mathrm{~b}$ ) from the input volume. Internal multiples were adapted, using progressively more aggressive filters. Subtraction was limited to the reflectors below the base of salt.

In the last example, water bottom and top salt are used as the main internal multiple generators. Several issues were addressed by the internal multiple attenuation. In Figure $6 a$, the oval in highlights an event that can be interpreted as an unconformity below the salt, while the rectangle shows two crossing reflectors. Figure $6 \mathrm{~b}$ shows internal multiples generated at the top of salt. In Figure $6 \mathrm{c}$ is the result of the adaptive summation, in which the possible unconformity is attenuated, the dipping reflectors are more continuous within the oval, and one of the crossing events is no longer present in the rectangle.

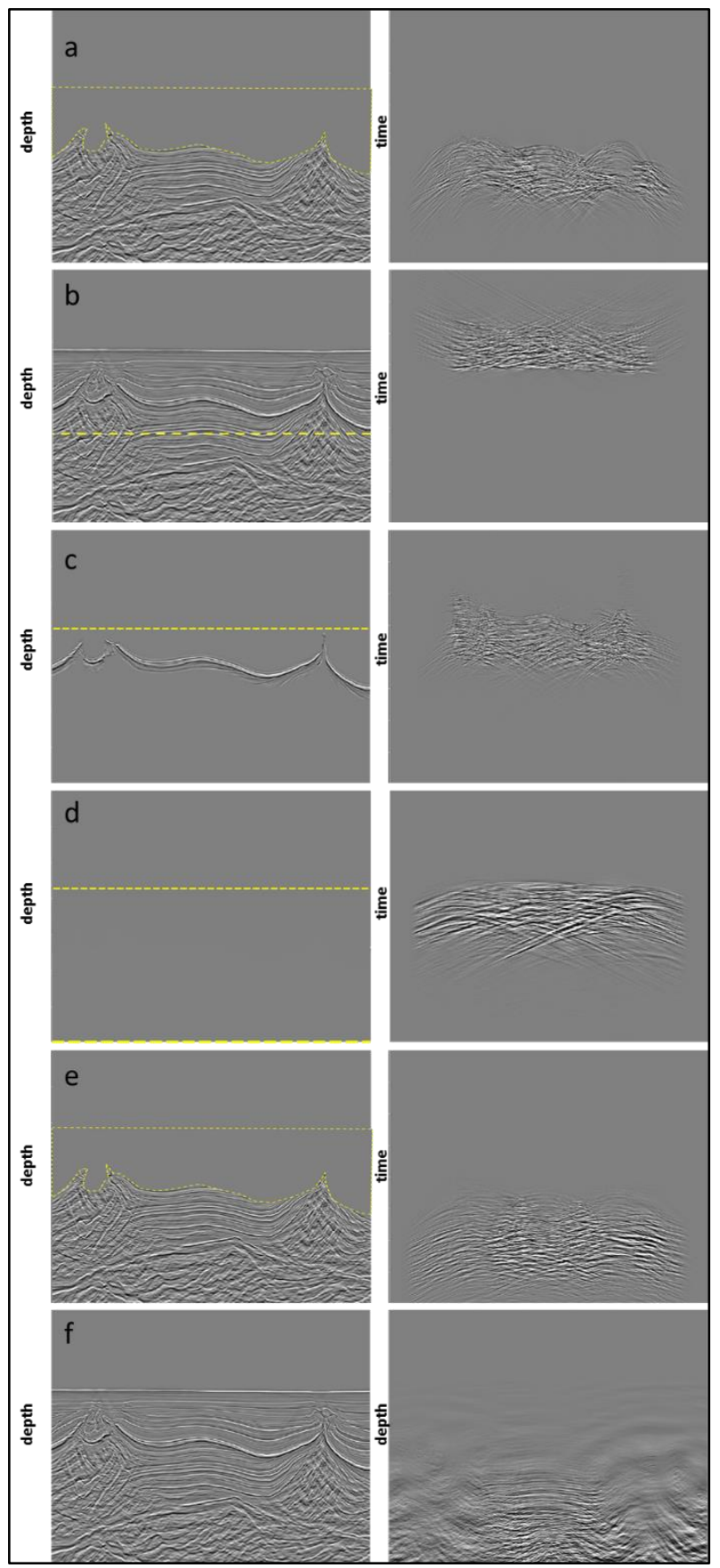

Figure 3- Illustration of the steps for modeling the postack-migrated-domain internal multiples. Letters correspond to the previously described steps.

In Figures $6 \mathrm{~d}$ and $6 \mathrm{e}$, are zoomed views of the Pre-salt after and before multiple attenuation, respectively. In Figure 7 , we can see the image before multiple attenuation (7a), after (7b), internal multiples generated at the water bottom (7c), and at the top of salt (7d). Notice how cleaner are the Pre-salt reflectors after attenuation. Also, some reflectors in the stratified salt were attenuated. Figure 8 shows an inline and a depth slice before (8a) and after (8b) 
multiple attenuation. In Figure $8 \mathrm{a}$, the arrow shows the directions of subtle events interfering with reflectors. Within the ovals, these events are no longer present after the internal multiple attenuation.

\section{Conclusions}

We introduce a method for modeling internal multiples in the postack-migrated domain. It applies when internal multiples are present in the final image, posing challenges to interpretation. The method is computationally inexpensive, since it uses the Exploding Reflector Model and oneway propagation. The examples shown attest its efficacy in attenuating internal multiples in $3 \mathrm{D}$ real data.

\section{Acknowledgments}

Authors would like to acknowledge Petrobras for allowing publishing this work.

\section{References}

Cypriano L., Marpeau F., Brasil R., Welter G., Prigent H., Douma H., Velasques M., Boechat J., Carvalho P., Guerra C., Theodoro C., Martini A., and Cruz J.N., 2015, Improved imaging of pre-salt targets in the Santos basin off-shore Brazil through attenuation of inter-bed multiples. 14th International Congress of the Brazilian Geophysical Society \& EXPOGEF, Rio de Janeiro, Brazil: 1262-1267.

Filpo E. and Tygel M., 1999, Deep-water multiple suppression in the near-offset range, The Leading Edge, 18,1 ,
Hembd J., Griffiths M., Ting C.O., and Chazalnoel N., 2010, Application of interbed multiple attenuation in the Santos Basin, Brazil. SEG Technical Program Expanded Abstracts: 3451-3455.

Krueger J., D. Donno, R. Pereira, D. Mondini, A. Souza, J. Espinoza, and A. Khalil, 2018, Internal multiple attenuation for four Pre-salt fields in the Santos Basin, Brazil. SEG Technical Program Expanded Abstracts: 4523-4527.

Loewenthal, D., L. Lu, R. Roberson, and J. Sherwood, 1976, The wave equation applied to migration: Geophysical Prospecting, 24, 380-399

Pica, A. and Delmas, L., 2008, Wave-equation based internal multiple modeling in 3D: 78th Annual International Meeting, SEG, Expanded Abstracts, 2476-2480.

Pica A. and Manin M., 2003, Fast zero-offset surface multiple modeling in 3D, SEG Technical Program Expanded Abstracts, 1957-1960

Staring M., Neut J., and Wapenaar K., 2018, Marchenko redatuming by adaptive double-focusing on $2 D$ and $3 D$ field data of the Santos basin. SEG Technical Program Expanded Abstracts: 5449-5453.

Wang B., Cai J., Guo M., Mason C., Gajawada S., and Epili D., 2011, Postmigration multiple prediction and removal in the depth domain. Geophysics, 76, WB217-223 

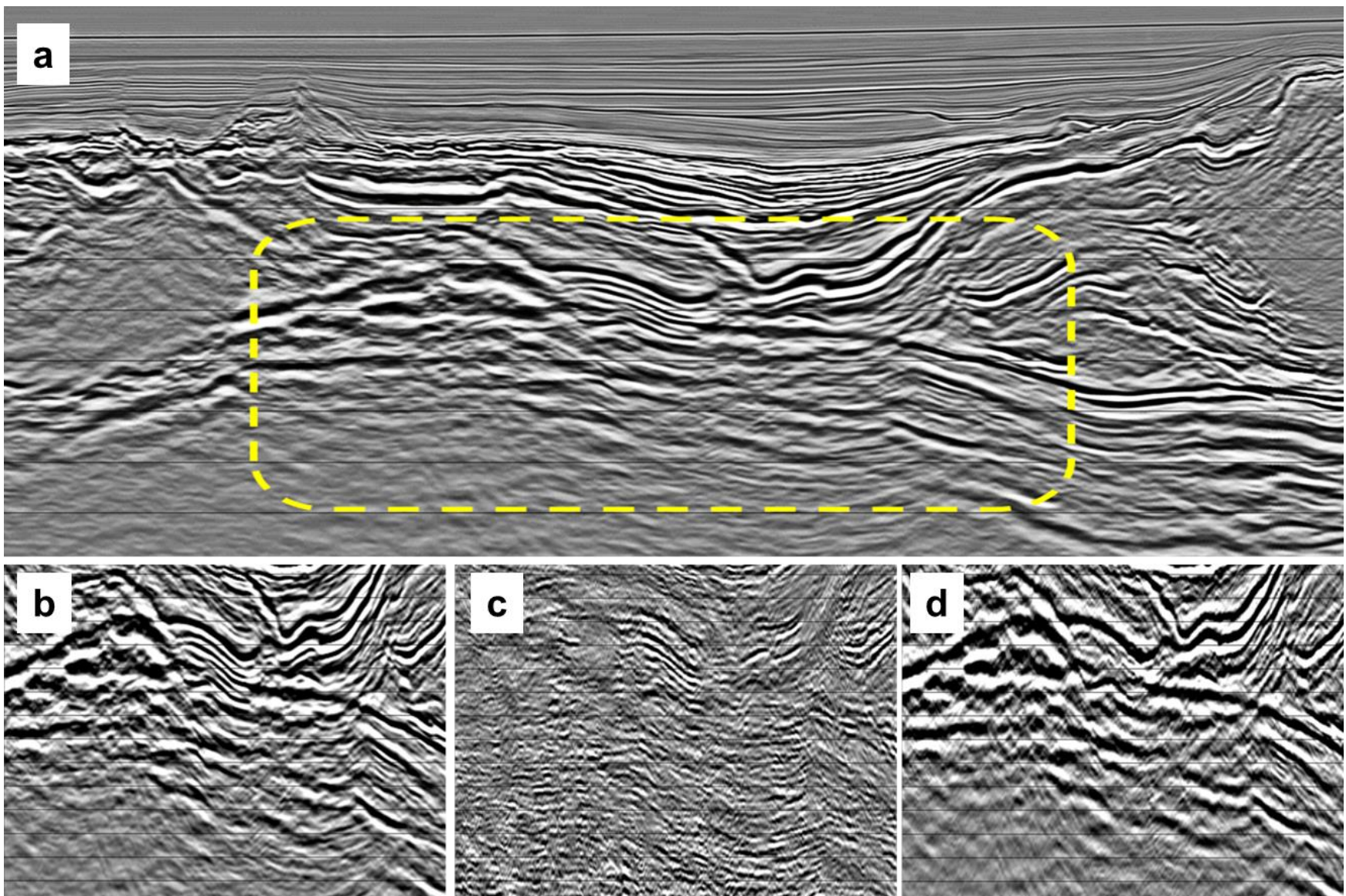

Figure 4 - The oval in (a) highlights the interference between steep reflectors and interbed multiples generated at the top of salt. Modeled internal multiples are represented in (b). In (c), one can observe the successful subtraction of the modeled multiples.
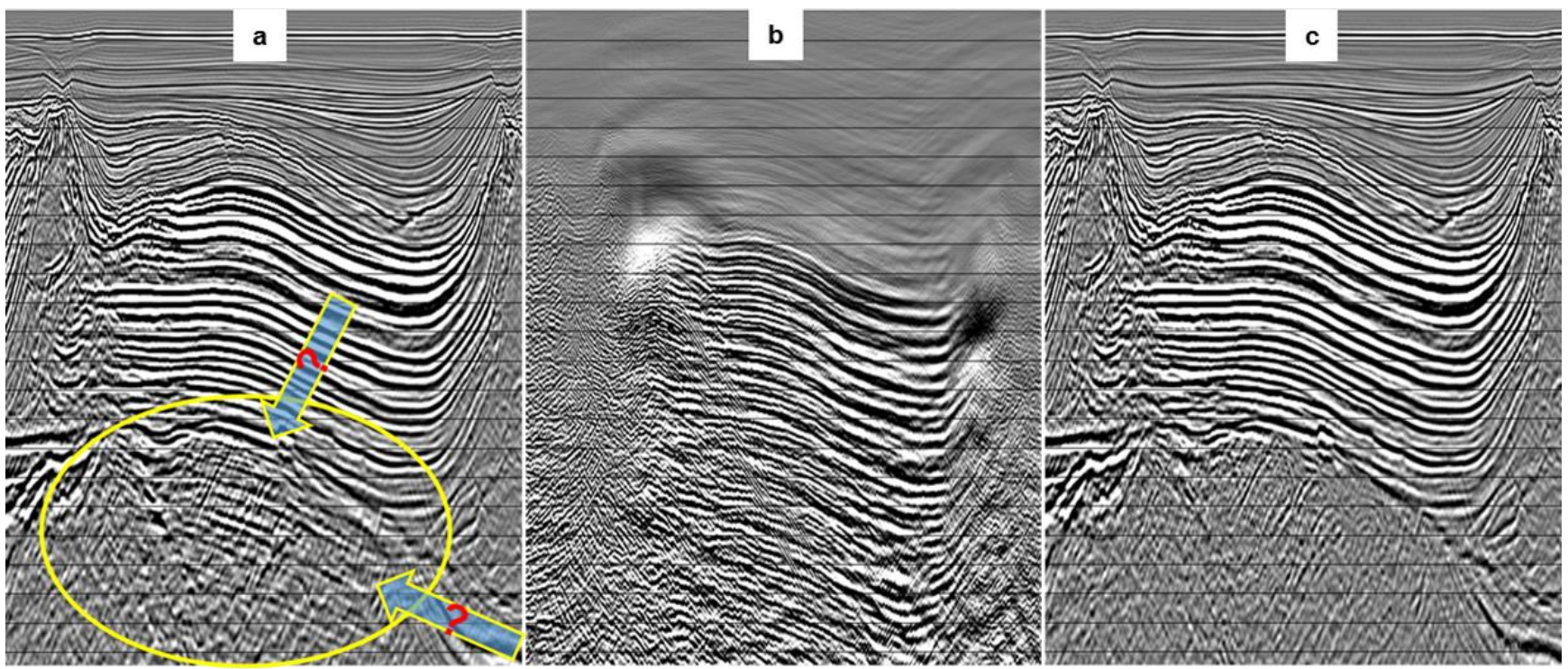

Figure 5 - The oval in (a) highlights the interference between steep reflectors and interbedded multiples generated at the top of salt. Modeled internal multiples are represented in (b). In (c), one can observe the successful subtraction of the modeled multiples. 

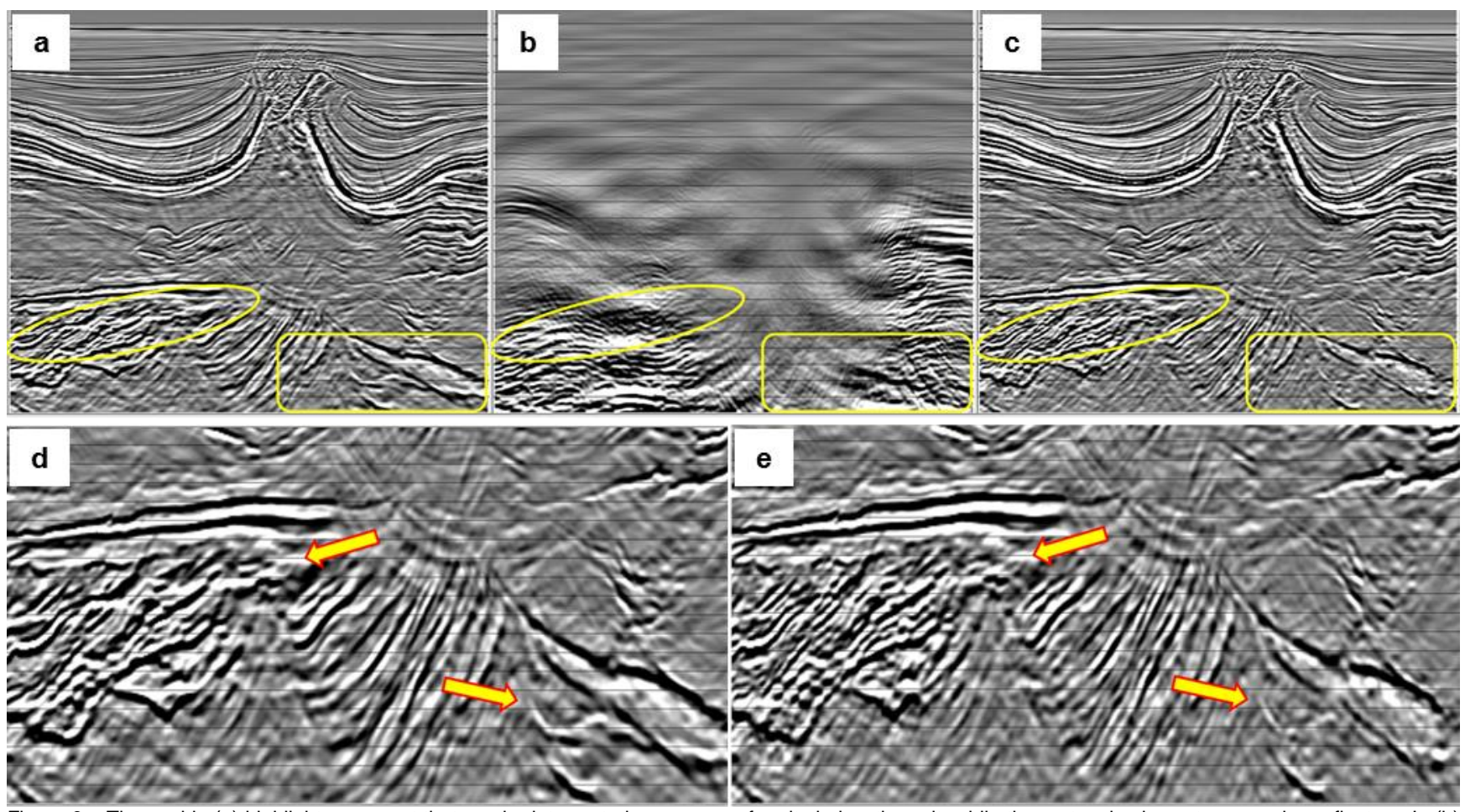

Figure 6 - The oval in (a) highlights an event that can be interpreted as an unconformity below the salt, while the rectangle shows two crossing reflectors. In (b) are represented internal multiples generated at the top of salt. In (c) is the result of the adaptive summation, in which the possible unconformity is attenuated and the dipping reflectors are more continuous within the oval and one of the crossing events is no longer present in the rectangle. In (d) and (e), are zoomed views of the Pre-salt after and before multiple attenuation, respectively.
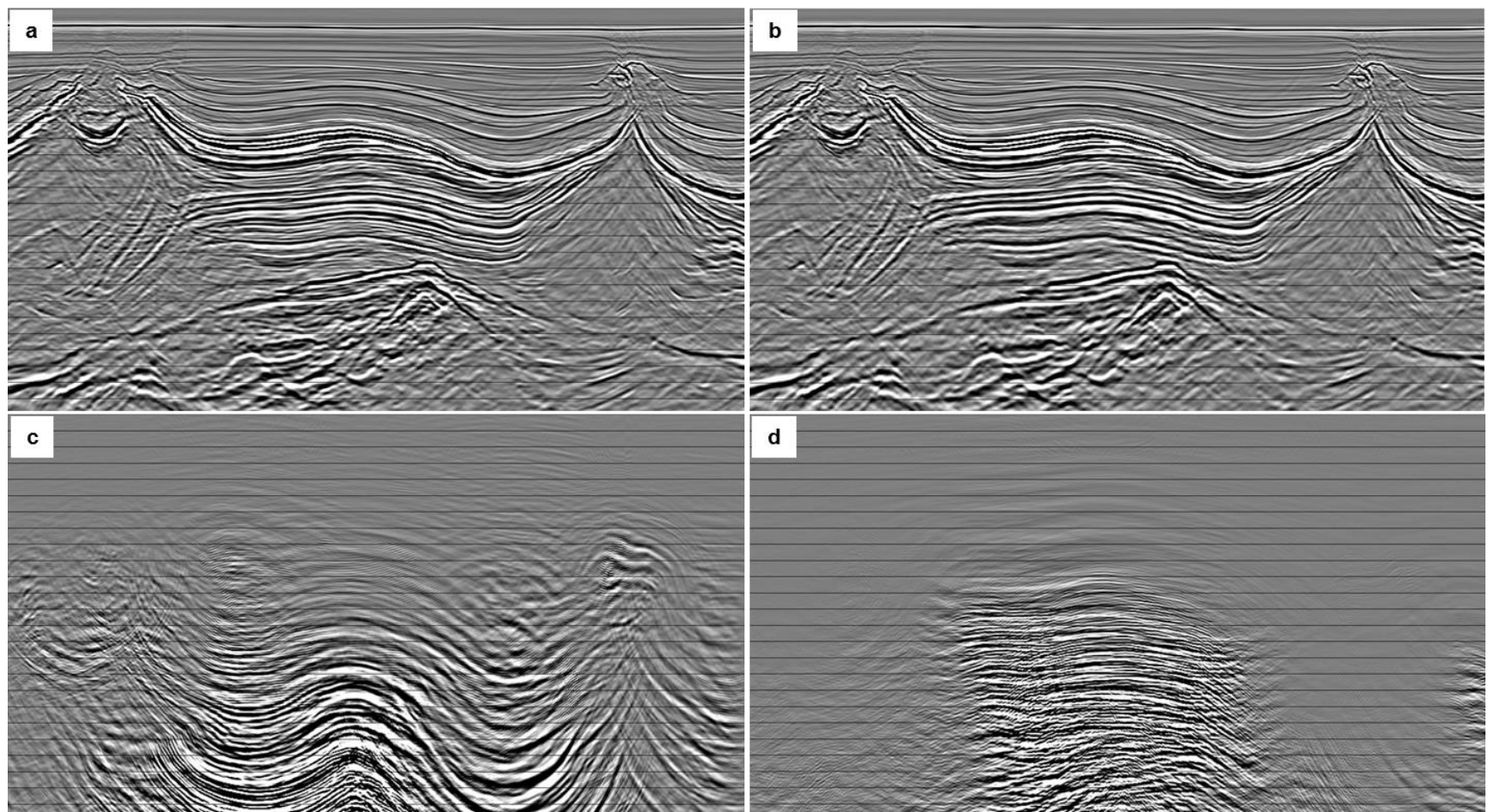

d

Figure 7 - Figures show the image betore multiple attenuation (a), after (b), internal multiples generated at the water bottom (c), and at the top of salt (d). Notice how cleaner is the image after multiple attenuation. 

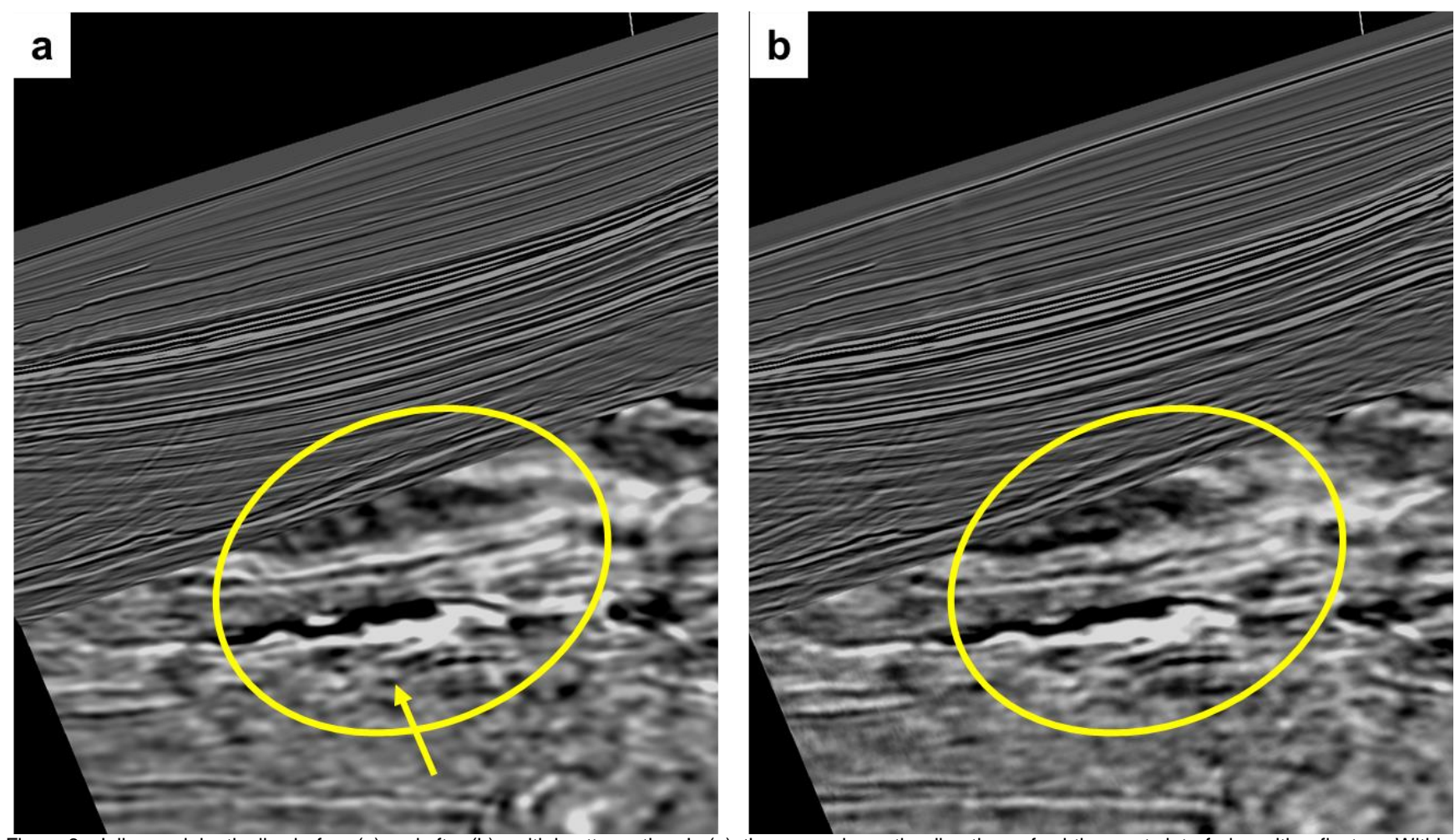

Figure 8 - Inline and depth slice before (a) and after (b) multiple attenuation. In (a), the arrow shows the directions of subtle events interfering with reflectors. Within the ovals, these events are no longer present after the internal multiple attenuation. 